\title{
OPEN Limited-angle computed tomography with deep image and physics priors
}

\author{
Semih Barutcu ${ }^{1 \bowtie}$, Selin Aslan ${ }^{2}$, Aggelos K. Katsaggelos ${ }^{1}$ \& Doğa Gürsoy ${ }^{1,2}$
}

Computed tomography is a well-established $\mathrm{x}$-ray imaging technique to reconstruct the threedimensional structure of objects. It has been used extensively in a variety of fields, from diagnostic imaging to materials and biological sciences. One major challenge in some applications, such as in electron or x-ray tomography systems, is that the projections cannot be gathered over all the angles due to the sample holder setup or shape of the sample. This results in an ill-posed problem called the limited angle reconstruction problem. Typical image reconstruction in this setup leads to distortion and artifacts, thereby hindering a quantitative evaluation of the results. To address this challenge, we use a generative model to effectively constrain the solution of a physics-based approach. Our approach is self-training that can iteratively learn the nonlinear mapping from partial projections to the scanned object. Because our approach combines the data likelihood and image prior terms into a single deep network, it is computationally tractable and improves performance through an end-toend training. We also complement our approach with total-variation regularization to handle highfrequency noise in reconstructions and implement a solver based on alternating direction method of multipliers. We present numerical results for various degrees of missing angle range and noise levels, which demonstrate the effectiveness of the proposed approach.

Computed tomography (CT) has found widespread applications in various areas from diagnostic imaging to studies of materials and biological systems ${ }^{1-7}$. In CT, an object illuminated by $\mathrm{x}$-rays is rotated while collecting projection image frames through a pixel array detector. In some CT setups, a uniform sampling of projections around the object is not possible due to the experimental setup or the extended shape of the object. For instance, in electron tomography, the angles are typically limited between -70 to $+70^{\circ}$. The challenge also appears for imaging planar objects such as integrated circuits because the light cannot penetrate the object due to increased thickness or absorption at certain angles. Sometimes, this problem is also referred to as the "missing wedge" problem since projections do not have any information about a wedge-shaped area in the Fourier space ${ }^{8}$; see Fig. 1 for the illustration of the problem.

There are optimization-based iterative approaches to compensate for the imaging artifacts induced by the limited projections. Algebraic reconstruction approaches, such as the algebraic reconstruction technique ${ }^{9}$, or the simultaneous iterative reconstruction technique ${ }^{10}$, often perform better than the traditional filtered-backprojection (FBP) method, at the expense of added computational cost. To better constrain the solution, compressive sensing techniques ${ }^{11,12}$ have been applied to promote sparsity in reconstructions. Total variation (TV) regularization is commonly used to obtain improved accuracy of the solution while suppressing high-frequency noise in reconstructions. However, a challenge is the choice of the regularization parameter, which is often performed heuristically in practical applications.

Learning-based approaches for improving the reconstructions provide an alternative. Most of the initial studies are essentially focused on image processing with pre-trained deep networks, either to in-paint sinogram images $^{13-16}$, or to correct artifacts in reconstructed images ${ }^{17,18}$. Therefore, they can be considered as a nonlinear filtering operation before or after the image reconstruction step. Although successful results have been achieved using pre-trained deep networks, the effectiveness of these approaches is fundamentally limited by the training data used. While these methods offer superior results in fields like medical imaging, where training data may be readily available, they are often not applicable to the fields of microscopy due to the limited amount of datasets for training.

A better approach for applications, for which training data is not available or scarce, is to use untrained networks, called deep image priors (DIPs) ${ }^{19}$. With DIPs, the network architecture itself constrains the solution.

${ }^{1}$ Northwestern University, 2145 Sheridan Road, Evanston, IL 60208, USA. ${ }^{2}$ Argonne National Laboratory, 9700 South Cass Avenue, Lemont, IL 60439, USA. ${ }^{\circledR}$ email: semihbarutcu@u.northwestern.edu 
(a) Conventional Tomography
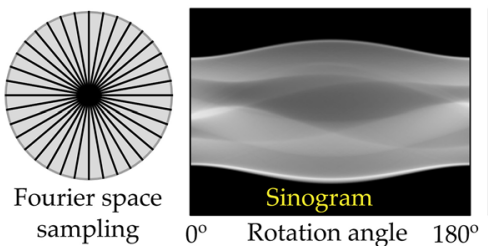

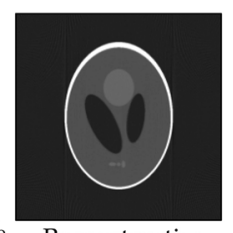

Reconstruction (b) Limited Angle Tomography

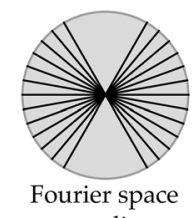

sampling

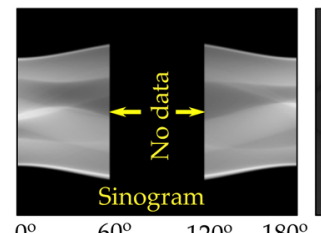

$120^{\circ}$

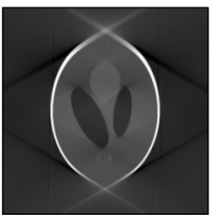

Reconstruction

Figure 1. Comparison of the conventional and limited angle tomography problems.

Similarly to the compressive sensing approach, DIPs can perform image processing tasks like inpainting or image recovery from incomplete observations. They do so by leveraging the superior low-dimensional image representation capability of deep generative networks. Recently, DIPs are proposed for the CT problem as a post-processing technique after tomographic reconstruction ${ }^{20}$. In a related study, DIP is used for the limited angle problem but for diffraction tomography ${ }^{21}$, where the measured images are in the Fourier space. In parallel, Yang et al. ${ }^{22}$ introduced a similar self-training optimization approach by combining DIP and tomographic reconstruction through a single network. While they showed great improvements compared to non-learning approaches, they also reported the instability of the method when measurements are noisy.

In this paper, we apply DIP to the limited angle problem for tomography, and extend DIP by incorporating regularization to improve robustness to noise. To effectively solve the problem, we also introduce an augmented Lagrangian type approach, which enables the use of the alternating direction method of multipliers (ADMM). Because with ADMM, we can break the imaging problem into smaller and well-defined sub-problems that can be solved independently, we can effectively map those sub-problems onto available computing resources. To further improve the method, we extend the work of Yang et al. ${ }^{22}$ by incorporating a fully-connected network before the DIP network, as the reconstruction is heavily dependent on the inverse projection operator, and a fully connected network can be trained as one. Through numerical experiments, we analyze the convergence behavior of the method and show that our approach can outperform standard methods like FBP and TV, based on the structural similarity index (SSIM) and peak signal-to-noise ratio (PSNR).

\section{Methods}

In this section, we present our approach to model the inverse problem of limited angle tomography and our solution approach. Our approach uses a generative model for constraining the solution, an approach similar to deep image priors (DIP) ${ }^{19}$. We also combine this approach with regularization methods for improved robustness of the reconstruction in noisy settings.

The inverse problem. The limited angle tomography acquisition process is well approximated by a linear imaging model as:

$$
d=R x+n
$$

where $R: \mathbb{R}^{M x 1} \rightarrow \mathbb{R}^{N x 1}$ is the x-ray projection operator that defines a mapping from a three-dimensional object represented in a vector form, $x \in \mathbb{R}^{M x 1}$, to a set of projection data, $d \in \mathbb{R}^{N x 1}$, with measurement noise, $n \in \mathbb{R}^{N x 1}$. A common approach to solve the inverse limited angle tomography problem, is to carry out the optimization:

$$
\hat{x}=\arg \min _{x}\|R x-d\|_{1}+\alpha T V(x)
$$

$T V$ is the total variation distance, and is defined as $T V(x)=\|\nabla x\|_{1}$. The first term is the data fidelity term measured by the $\ell_{1}$ norm and the second term is the regularization term to favor piecewise-constant solutions. $\lambda$ is the regularization parameter that controls the trade-off between these terms. $\ell_{1}$ norm is chosen for data fidelity term due to the increased reconstruction quality compared to $\ell_{2}$ norm at early experimentation.

To leverage deep generative models for effectively constraining the solution, we will describe $x$ with, $G_{w}: \mathbb{R}^{N x 1} \rightarrow \mathbb{R}^{M x 1}$, where $w$ represents the weights of the deep neural network. This leads to the optimization problem of the form:

$$
\hat{w}=\arg \min _{w}\left\|R G_{w}(d)-d\right\|_{1}+\alpha\left\|\nabla G_{w}(d)\right\|_{1} .
$$

Because the network defines a mapping from the data domain to the object domain, it can be seen as an approximation to the inverse $\mathrm{x}$-ray projection operation. Therefore, it captures both attributes of physics-based inversion and deep image prior into a single deep neural network.

Solution of the inverse problem. For solving Eq. (3), we first convert the problem into a constrained optimization problem by using an auxiliary variable, and use the alternating direction method of multipliers $(\mathrm{ADMM})$ for the solution ${ }^{24}$. The constrained problem formulation is given as:

$$
\hat{w}, \hat{y}=\arg \min _{w, y}\left\|R G_{w}(d)-d\right\|_{1}+\alpha\|y\|_{1} \quad \text { subject to } \nabla G_{w}(d)=y
$$

and the corresponding augmented Lagrangian is written as follows, 


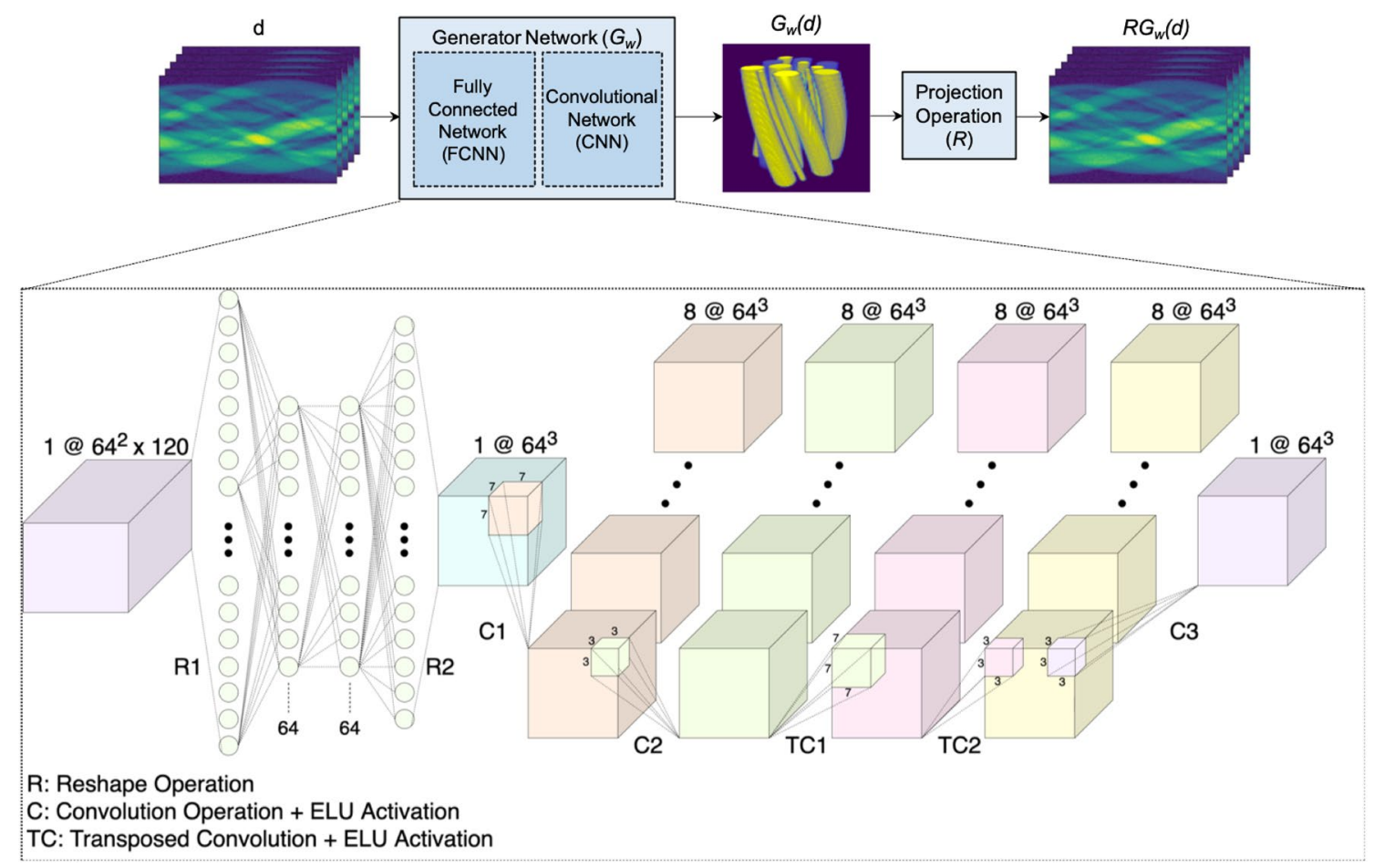

Figure 2. Illustration of the operators and deep neural network architecture ${ }^{23}$.

$$
L(w, y, z)=\left\|R G_{w}(d)-d\right\|_{1}+\alpha\|y\|_{1}+z^{T}\left(\nabla G_{w}(d)-y\right)+\tau / 2\left\|\nabla G_{w}(d)-y\right\|_{2}^{2},
$$

where $\tau>0$ is an auxiliary parameter and $z \in \mathbb{R}^{N x 1}$ is introduced as the dual variable. We use ADMM to minimize Eq. (5) by iteratively solving for $w$ and $y$, followed by the update of the dual variable $z$, that is,

$$
\begin{gathered}
w^{k+1}=\arg \min _{w}\left\{\left\|R G_{w}(d)-d\right\|_{1}+\tau / 2\left\|\nabla G_{w}(d)-y^{k}+z^{k} / \tau\right\|_{2}^{2}\right\} \\
y^{k+1}=\arg \min _{y}\left\{\alpha\|y\|_{1}+\tau / 2\left\|\nabla G_{w^{k+1}}(d)-y+z^{k} / \tau\right\|_{2}^{2}\right\} \\
z^{k+1}=z^{k}+\tau\left(\nabla G_{w^{k+1}}(d)-y^{k+1}\right) .
\end{gathered}
$$

At each ADMM iteration, $L(w, y, z)$ is minimized over each variable by fixing the other variables until convergence.

The operators for the problem and the network architecture architecture are illustrated in Fig. 2. Because the deep generative network in sub-problem Eq. (6) is used to both represent the inverse x-ray projection operation and the deep image prior, we use two cascaded neural network architectures: a fully connected network followed by a convolutional network. In this configuration, the input projections are mapped to the reconstruction domain by the fully connected layers, and then they are converted to a three-dimensional array. Convolutional layers then convert the features of the $3 \mathrm{D}$ tensors to minimize sub-problem Eq. (6).

The proposed solution utilizes the deep image priors in order to improve the reconstruction. The networks implicitly optimize the weight for inpainting missing parts of the projections with the help of the physical forward model. Although there is missing information, low-level statistical distribution learning is used to estimate the $3 \mathrm{D}$ object structure, and the network reconstructs the $3 \mathrm{D}$ object by filling out the missing information in the measurements. The capability of the network to inpaint the missing parts of the projections depends on the convergence of the self-learning process to optimize the weights of the network. 


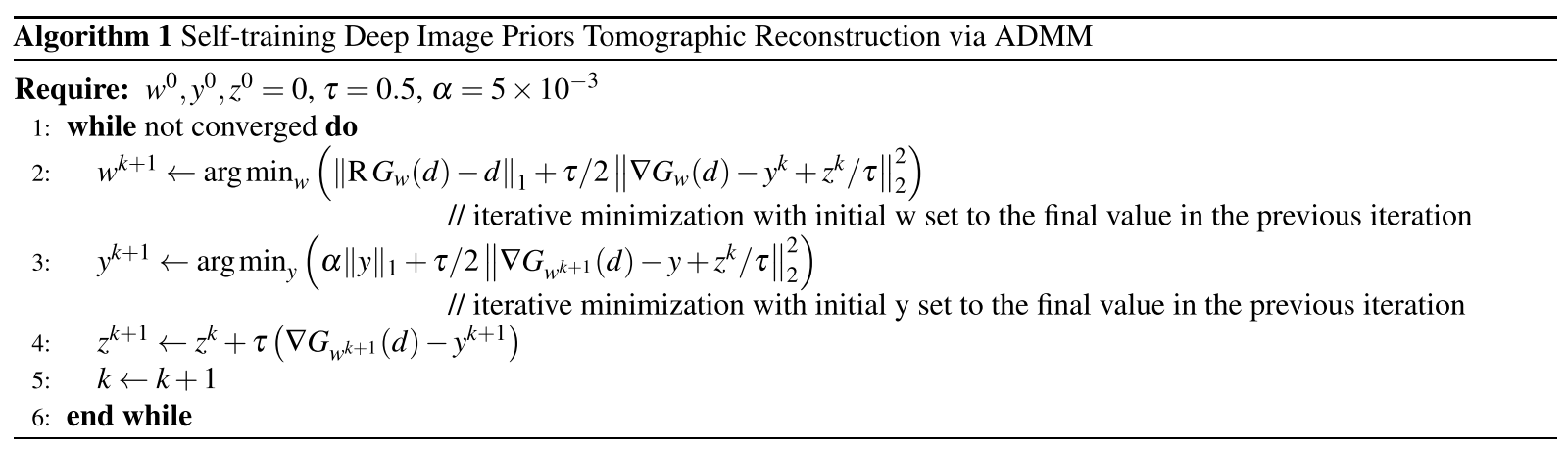

Implementation details. Cascaded architecture of the generative network is shown in Fig. 2 for an input of 120 projections acquired from an object with a support of $64 \times 64 \times 64$ pixels. First part of the network consists of four fully-connected layers where hidden layers have 64 nodes each. Each fully-connected layer is followed by a hyperbolic tangent activation function, a layer normalization operation, and a dropout layer with a dropout ratio of 0.25 . Then, the output of the fully-connected network is reshaped into $64 \times 64 \times 64$. The following convolutional network consists of two 3D convolutional layers, two 3D transposed convolutional layers, and one 3D convolutional layer with kernel sizes of 7, 3, 7, 3, and 3, respectively. Padding is applied for maintaining the size and stride size is kept at 1 , while 8 filters are used for each convolutional layer. Similarly, each convolutional layer is followed by a Exponential Linear Unit (ELU) activation and a layer normalization operation, and the output of the last convolutional layer is the reconstruction of $3 \mathrm{D}$ object.

Algorithm 1 presents the pseudo-code of our ADMM algorithm. For the minimization of sub-problem Eq. (6), we use the Adam optimizer ${ }^{25}$ with a learning rate of $2 \times 10^{-5}$, and the two momentum terms are equal to 0.5 and 0.999 . We experimented with different values for the total variation regularizer parameter $\alpha$ empirically, and we found that values between $1 e-3$ and $1 e-5$ give reasonable results for different objects and different levels of desired piece-wise smoothness. This change depends on the object, and the reconstruction is not too sensitive to change of the value of $\alpha$. We initialize $w, y$, and $z$ with zeros at the first iteration, and each of the inner optimizations for w [Eq. (6)] and y [Eq. (7)] are initialized with their corresponding values from their converging values at the previous outer iterations. tau is initialized with the value of 0.5, and it is updated based on Eqs. (9), (10) and (11 )at every outer iteration for optimizing the value for the best convergence behavior. The number of iterations for estimating the network weights is set to 1000 for each ADMM iteration. Similarly to other DIP implementations, we use early stopping as a regularization to avoid over-fitting the noise.

$$
\begin{gathered}
\epsilon_{1}^{k}=\left\|\nabla G_{w^{k}}(d)-y^{k}\right\|_{2} \\
\epsilon_{2}^{k}=\tau^{k}\left\|\nabla G_{w^{k}}(d)-\nabla G_{w^{k-1}}(d)\right\|_{2} \\
\tau^{k+1}= \begin{cases}2 \tau^{k}, & \text { if } \epsilon_{1} \geq 10 \epsilon_{2} \\
\frac{1}{2} \tau^{k}, & \text { if } \epsilon_{2} \geq 10 \epsilon_{1}\end{cases}
\end{gathered}
$$

We use soft-thresholding ${ }^{26}$ for explicitly solving the subproblem Eq. (7). For simplicity, we assume element-wise operations for taking the absolute value, comparing to zero, and dividing. the resulting update for $y$ is:

$$
y^{k+1}=\frac{\nabla G_{w^{k+1}}(d)+z^{k} / \tau}{\left|\nabla G_{w^{k+1}}(d)+z^{k} / \tau\right|} \max \left\{0,\left|\nabla G_{w^{k+1}}(d)+z^{k} / \tau\right|-\alpha / \tau\right\} .
$$

We normalize the range of input variables to avoid overflowing of gradients. The normalization is done in two steps: first, $\mathrm{z}$-score normalization is done to maintain a zero mean and unit variance; and second, $\min$-max normalization is done to obtain values between 0 and 1 . We apply normalization both to the input and the computed projections. In other words, we normalize the x-ray transform operator, R. Besides, we apply a layer normalization ${ }^{27}$ to every layer of the network to improve the convergence and accuracy of the network.

\section{Results}

Simulation setup. To evaluate the effectiveness of the proposed method against the state-of-the-art methods, numerical tests are performed on two simulated phantoms with support of $64 \times 64 \times 64$ pixels. One phantom is a simulated $3 \mathrm{D}$ object consisting of two spiral-shaped materials created by XDesign ${ }^{29}$, that is made of a strongly absorbing material, gold $(\mathrm{Au})$, and a weakly absorbing material, zinc oxide ( $\mathrm{ZnO})$. The densities of these materials are 19.32 and $5.606 \mathrm{~g} / \mathrm{cm}^{3}$, respectively. A $5 \mathrm{keV}$ energy beam is used to simulate the refractive index values. The XDesign Phantom and its center slice are illustrated in Fig. 3a. The second phantom is chosen to be the 3D Shepp-Logan phantom from TomoPy ${ }^{30}$; see Fig. 3b for the illustration of the bottom half of the 3D object and its center slice. The objects are chosen to be sparse in $3 \mathrm{D}$ space to observe the artifact removal in limited angle tomography reconstructions more clearly. 
(a) 3D XDesign phantom and its center slice

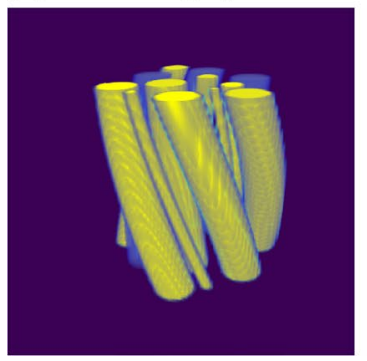

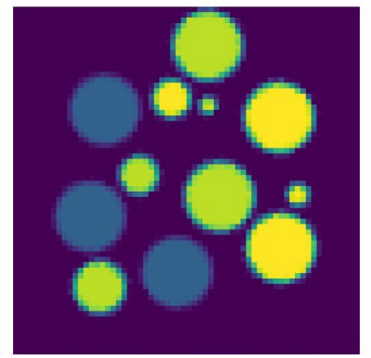

\section{(b) 3D Shepp-Logan phantom and its center slice}
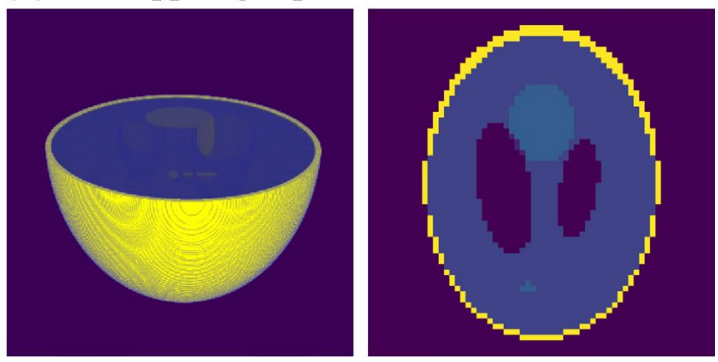

Figure 3. Simulated $3 \mathrm{D}$ objects ${ }^{23,28}$ and their center slices.

For simulations throughout the paper, projections are acquired via a limited angle tomography setup where the objects are rotated around their common axes. Projections are simulated by $1^{\circ}$ separation in the limited ranges of $0^{\circ}-120^{\circ}$ and $0^{\circ}-150^{\circ}$; corresponding to $60^{\circ}$ and $30^{\circ}$ of missing wedge information, respectively. In addition, Gaussian noise with increasing variance is added to test the noise removal capabilities of the proposed method, resulting in projections that have noise with variances $0.5,2.5,5$, and 10 . We assumed Gaussian noise on measurements because we mainly target transmission-type microscopes, where the photon fluence on the sample is reasonably high. However, for microscopes that use secondary signals like fluorescence emission or gamma radiation, a Poisson data formation model may be a better choice.

Analysis of convergence behavior. In this section, we discuss the convergence behavior of the proposed algorithm by evaluating how different losses behave as a function of each iteration. In Fig. 4a, we show the term $\left\|R G_{w^{k}}(d)-d\right\|_{1}$ as a function of iteration $\mathrm{k}$. It is a measure of how well the generated projections based on the reconstructed $3 \mathrm{D}$ object by the deep neural network fit the available data, for different number of projections and amounts of noise.

$$
\epsilon_{1}=\left\|R G_{w^{k}}(d)-d\right\|_{1}
$$

The amount of noise in the projections is the main factor determining the convergence of the projection fit. Although all setups are able to minimize this loss with increasing number of iterations, the error decreases to a significantly lower value in noise-free cases because it is not possible for the generated projections to fit Gaussian noise. This behavior is not only expected but also desired since the targeted reconstructions are required to be free of noise. Similarly, the error minimization of projection fit for noisy setups is adjusted by the regularization loss. Unlike the effect of noise, the effect of the missing wedge information is minimal on the convergence. With increased number of projection angles, the decay of the error term is slightly improved.

The second loss function to update the deep network is the loss derived from the regularization term. The auxiliary ADMM variable, $y$, which should converge to the gradient of a noise free reconstruction, is updated using total variation (TV) regularization to have minimum values except at the edges. Then, the network weights are updated by minimizing:

$$
\epsilon_{2}=\tau / 2\left\|\nabla G_{w}^{k}(d)-y^{k}+z^{k} / \tau\right\|_{2}^{2}
$$

As shown in Fig. 4b, unlike the convergence of the projection fit loss, change in the error amount of the regularization loss has a positive correlation with the variance of the noise in the projections. Since the target is for the reconstruction to have piece-wise smoothness, reconstructions with high variance are effected by regularization more, and the error is minimized accordingly for noisy projections. A trade-off between the effects of regularization and the projection fit is apparent in noisy projection setups, where increased piece-wise smoothness means worse fit to the sinograms. Due to this trade-off, decay curves show an increase in error for the regularization term with the increased number of iterations while the generated projections converge to the noisy projections. Similarly to the projection fit decay curve, the error for regularization also achieves smaller values for increased number of projections with decreasing effects of missing wedge artifacts.

ADMM convergence can be analyzed by the observation of the decay curves of primal and dual residuals of the ADMM framework. The primal and dual residuals, $r^{k+1}$ and $s^{k+1}$, respectively, can be derived from Eq. (5) as follows, where $\mathrm{k}$ is the outer iteration number:

$$
\begin{gathered}
r^{k+1}=y^{k+1}-\nabla G_{w^{k+1}}(d) \\
\left.s^{k+1}=\tau\left\{\nabla G_{w^{k+1}}(d)-\nabla G_{w^{k}}(d)\right)\right\}
\end{gathered}
$$

The derived primal and dual residuals demonstrate the behavior of the update steps taken in the ADMM iterations. While the primal residual is a measure of how well the reconstructions converge towards the auxiliary variable $z$, the dual residual shows how much the reconstruction is updated towards the solution. While Figs. 4c and demonstrates that residuals are minimized with increased number of iterations for each setup, the amount of decrease is more for the noisy reconstructions due to the increased divergence from the initial reconstructions and higher number of degrees of freedom they have. 
(a) Decay Curves of Projection Fit Losses

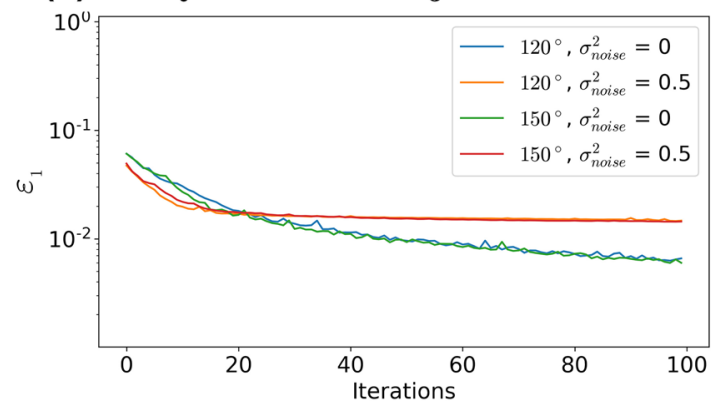

(c) Decay Curves of Primal Residuals

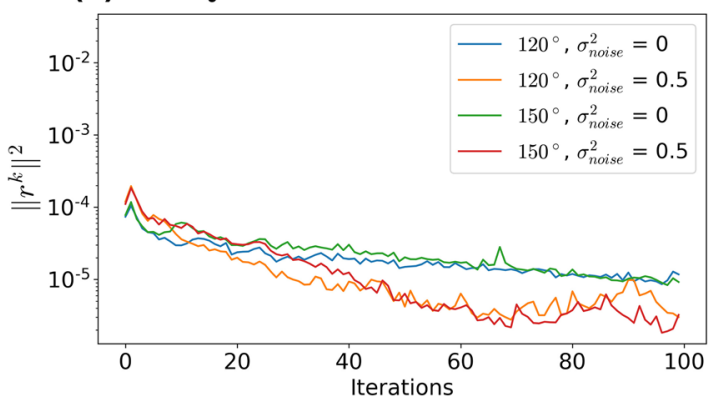

(b) Decay Curves of Regularization Losses

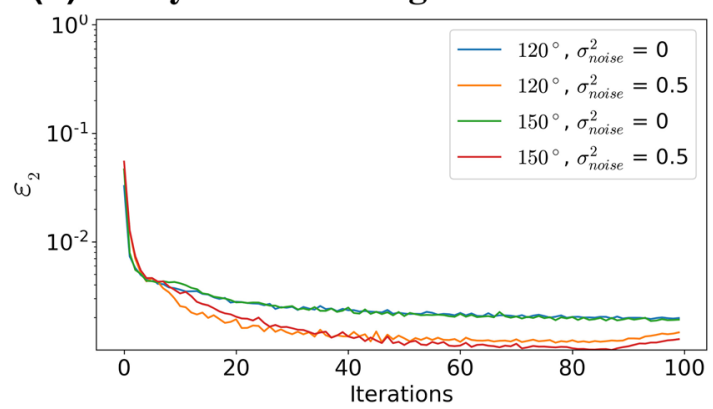

(d) Decay Curves of Dual Residuals

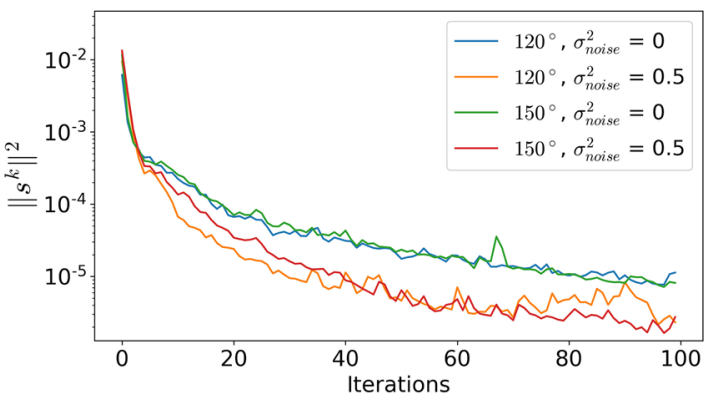

Figure 4. Convergence curve plots.

Reconstructions and comparisons. In this section, we show the reconstructions for different tomographic angle ranges, list the methods in the literature to be compared to the proposed approach, present the reconstructions for the simulations for all methods, and discuss the results qualitatively and quantitatively.

In Fig. 5, the center slices of 3D reconstructions of the two simulated objects with different tomographic scan angles in the noise-free case are demonstrated. As can be seen, reconstruction for $0^{\circ}-150^{\circ}$ case is the reconstruction is almost identical with the full scan angle case, and the degradation in the reconstruction for $0^{\circ}-120^{\circ}$ case is minimal with some small visible artifacts. With the increased missing wedge size, the reconstruction quality decreases as expected. However, the reconstruction for the Shepp-Logan phantom, which is a more compact object, is quite reasonable even for even the $0^{\circ}-40^{\circ}$ case, where the reconstruction for the $0^{\circ}-80^{\circ}$ does not even show easily visible artifacts. In contrast, the limitation of the approach for a more complex object such as the XDesign phantom can be observed for small tomographic angle range reconstructions. While the reconstruction for the $0^{\circ}-80^{\circ}$ case is acceptable, the structure of the object is lost in the $0^{\circ}-40^{\circ}$ case. In addition to the reconstructions in Fig. 5, reconstructions of a slab-shaped 2D object with square inclusions are provided in Supplementary Fig. S2 to show the effectiveness of the algorithm for different types of objects. It is observed that the proposed method also produces successful reconstructions with a slab-shaped object.

Comparative studies. To evaluate the improvements brought by the proposed method over the ones in the literature, reconstructions from the proposed algorithm are compared to the reconstructions from state-of-the-art algorithms. The baseline algorithm used for comparison is called GridRec ${ }^{31,32}$, a simple filtered-back projection method $^{33}$ implemented by the application of interpolation in Cartesian coordinates instead of polar coordinates. GridRec is demonstrated as the primal reconstruction method that does not take any steps to compensate for missing wedge artifacts or noise. A more elaborate and advanced algorithm is developed by Yang et al. ${ }^{22}$, deploying a self-training approach with a Generative Adversarial Network (GAN), called GANrec, and aiming at accurate slice-by-slice reconstructions by the use of a generative network with the help of a discriminator network loss. Although the method is successful for reconstructions in noise-free cases and eliminates some of the missing wedge artifacts, it is not robust to noisy projection inputs.

To understand the effectiveness of the noise removal by regularization in the proposed approach, a Total Variation (TV) reconstruction algorithm ${ }^{34}$ is used, which deploys a total variation regularization integrated into back-projection algorithm to lower the noise and missing wedge artifacts. For TV, an efficient implementation called TIGRE ${ }^{35}$ is used for reconstructions. The Deep Image Prior (DIP) algorithm is also tested by designing a DIP network as shown in Supplementary Fig. S1. The convolutional network implemented in DIP is chosen to be deeper than the cascaded network in the proposed model, and has the same number of trainable parameters. The network is trained in the same ADMM framework with the addition of total variation regularization. For GridRec, GANrec, TV, DIP, and the proposed method, the same forward projector ${ }^{36}$ is used, and GANrec is implemented in PyTorch for a fair comparison.

Comparisons. In Fig. 6, center slices of the 3D reconstructions of GridRec, GANrec, TV, Deep Image Priors with TV (DIP-TV), and the proposed method (TomoDIP-TV) from noise-free projections are shown. For 


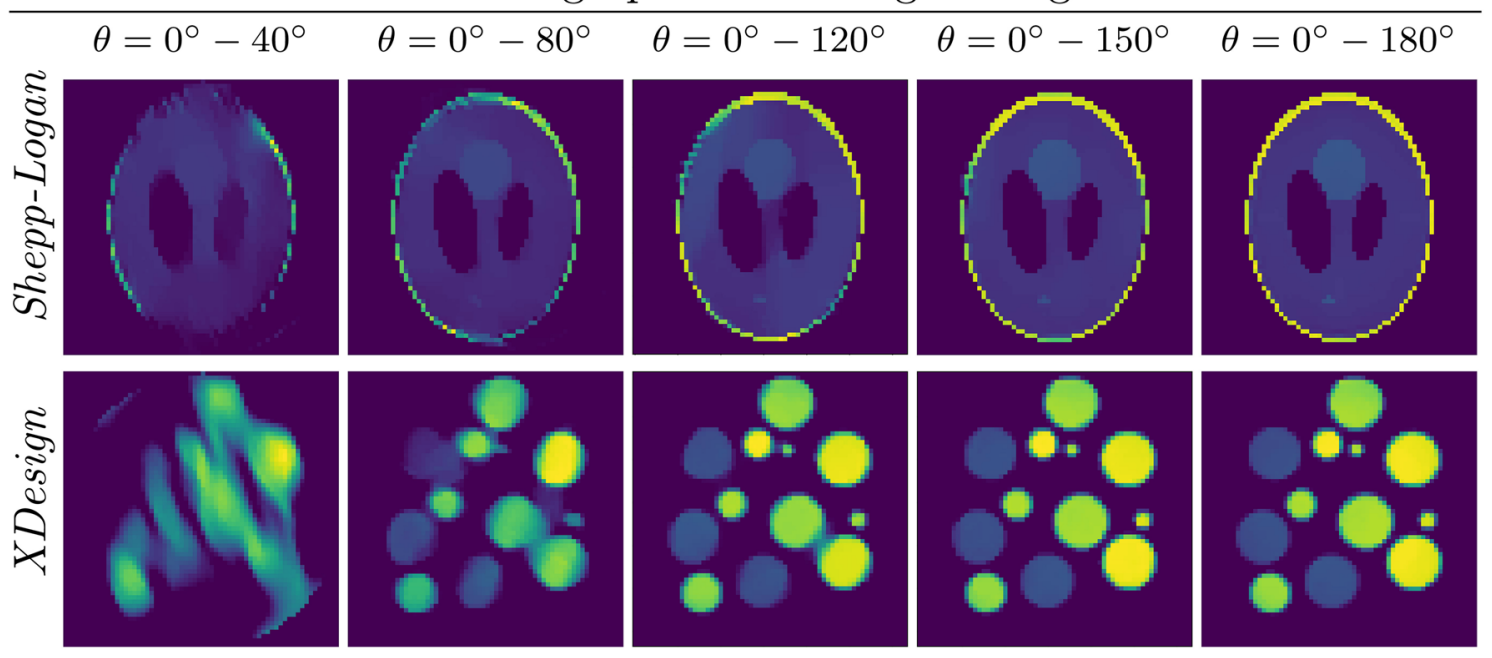

Figure 5. Reconstructions for projection in varying angles from noise-free projections.

both angle ranges and phantoms, GridRec fails to generate comparable results due to the missing projections. Reconstructions from GANrec are significantly improved compared to the GridRec. GANrec is able to eliminate elongation artifacts at the edges of the sections of the object, although it shows some streaking artifacts in the areas that should have been piece-wise constant. Details of the improvements at the edges are more visible in the zoomed images; however, streaking artifacts are shown to be above the desired limits for the low number of projections. This effect is more observable for the XDesign phantom. TV method improves the piece-wise smoothness with the help of regularization, but fails at compensating for missing wedge artifacts. As shown in the zoomed images, although the streaking artifacts are reduced, borders of the objects experience notable artifacts. Besides, even though the reconstructions for fewer missing wedge data $\left(0^{\circ}-150^{\circ}\right)$ are close to the ground truth, effects of over-regularization can be seen at the edges. However, DIP-TV greatly reduces the artifacts and reconstructs the objects more accurately. The proposed method improves the reconstruction quality even more by reducing the artifacts at the edges to very low levels. This effect can be observed in detail in the zoomed images.

In Table 1, Structural Similarity Index Measure (SSIM) and Peak Signal-to-Noise Ratio (PSNR) for the noisefree reconstructions are shown. The quantitative measures of the reconstructions mostly align with observations in the qualitative analysis, and the proposed method performs the best overall. On the other hand, in particular setups, DIP-TV method generates slightly higher measures when there is enough data and less variance in the object values. While GridRec has the worst measures, GANrec and TV show their advantages in different setups.

The effect of increased noise variance in the projections is evaluated in Fig. 7 where center slices of the 3D reconstructions using GridRec, GANrec, TV, DIP-TV, and TomoDIP-TV from noisy projections are shown. For the simulations, the variance of the Gaussian noise is varied between 0.5 and 10 , while the projection angle range is kept the same $\left(0^{\circ}-150^{\circ}\right)$. As expected, GridRec and GANrec do not perform well when the input projections are noisy. In fact, GANrec suffers more from the increased noise since there is no mechanism in the algorithm to prevent the generated projections from fitting the noisy sinograms, creating even noisier reconstructions. TV performs significantly better than the previous two methods, but it suffers from poor reconstruction quality at the edges. Moreover, the reconstruction quality is greatly reduced with increased noise, where the trade-off between missing wedge artifacts and regularization is more apparent. DIP-TV takes advantage of the regularization effect of the deep image priors and reconstructs the $3 \mathrm{D}$ object well, even for the setups with high noise variance. However, it fails to eliminate artifacts to recover the object structure when the noise variance is increased beyond its limits. On the other hand, TomoDIP-TV takes advantage of the fully connected network to reconstruct the object structure and improves the quality with the help of DIP and TV regularization. Although the reconstructions for projections with high noise variance are far from ideal, the object structure is preserved more compared to the previous methods.

Quantitative analysis of the 3D reconstructions from noisy projections can be seen in Table 2. Most of the SSIM and PSNR values are consistent with the qualitative observations and highly favor the proposed method as the best reconstruction method. While reconstructions using GANrec have the lowest SSIM and PSNR values, the increase towards the proposed method in SSIM is notably higher than the increase in PSNR. In some cases, the PSNR values are slightly lower for the proposed method, whereas the SSIM values are significantly higher. For those setups, qualitative evaluations of the reconstructions in Fig. 7 show that even though the PSNR is lower, the structural integrity of the reconstructions is higher for the proposed method than for the rest of the algorithms.

Although the sinogram completion effect of the DIP method is not explicit, it can be observed in sinograms of the reconstructed objects. A comparison of the complete sinogram for the Shepp-Logan phantom and the reconstructed sinogram for the noise-free case is given in Fig. 8. As observed, the missing part of the sinogram is constructed using only the information from the other parts of the sinogram, which do not necessarily give straightforward information about the missing part. This difference shows the advantage of the DIP method over the techniques in the literature, which lack the ability to complete the sinogram information in detail. 


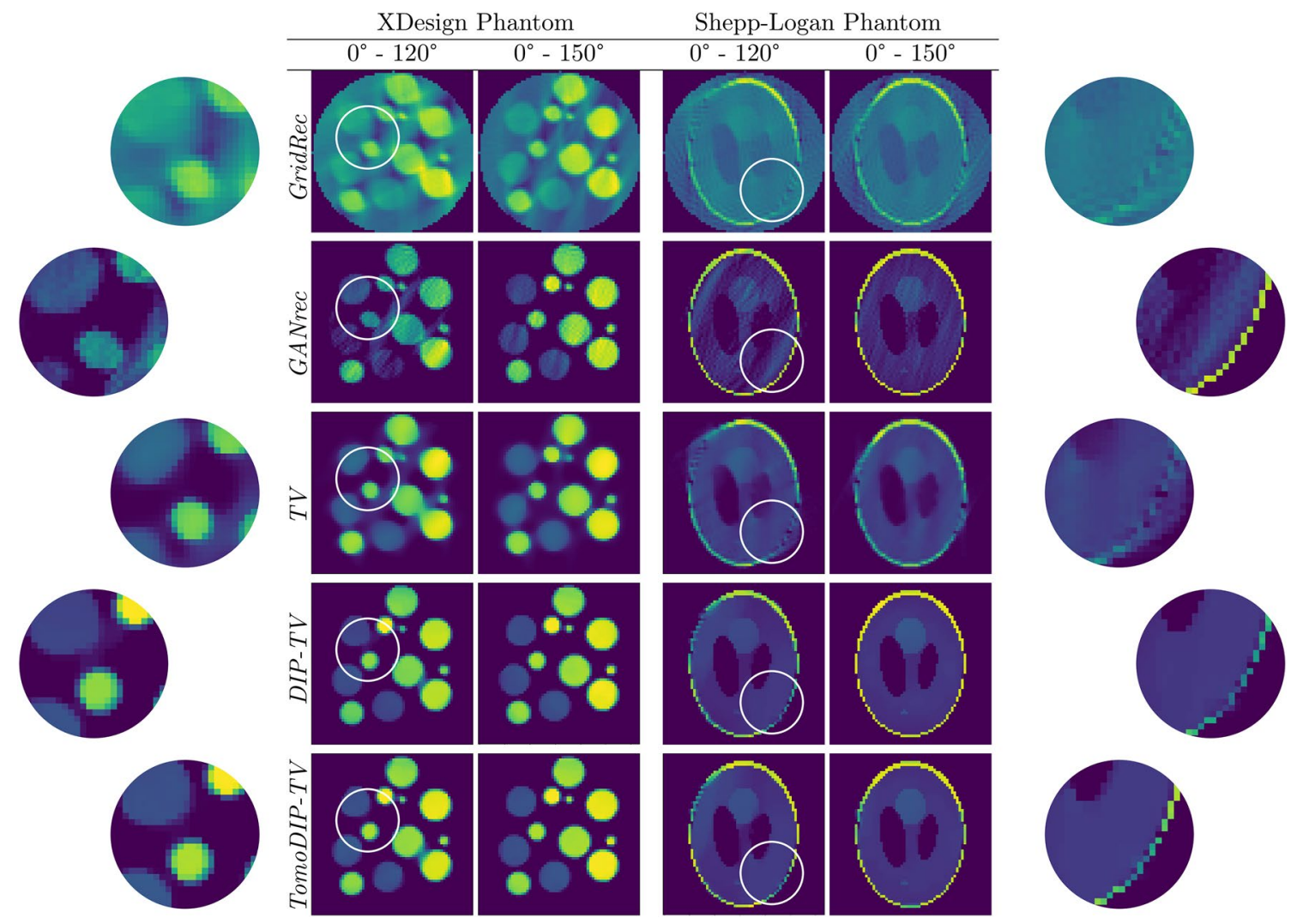

Figure 6. Reconstruction comparison for the XDesign phantom and the Shepp-Logan phantom for the noisefree case.

\begin{tabular}{|c|c|c|c|c|c|c|c|c|}
\hline \multirow[b]{3}{*}{ 3D Noise free reconstruction } & \multicolumn{4}{|c|}{ XDesign phantom } & \multicolumn{4}{|c|}{ Shepp-Logan phantom } \\
\hline & \multicolumn{2}{|c|}{$0^{\circ}-120^{\circ}$} & \multicolumn{2}{|c|}{$0^{\circ}-150^{\circ}$} & \multicolumn{2}{|c|}{$0^{\circ}-120^{\circ}$} & \multicolumn{2}{|c|}{$0^{\circ}-150^{\circ}$} \\
\hline & ssim & psnr & ssim & psnr & ssim & psnr & ssim & psnr \\
\hline GridRec & 0.49 & 60.90 & 0.56 & 62.50 & 0.39 & 62.51 & 0.42 & 63.20 \\
\hline GANrec & 0.74 & 65.76 & 0.90 & 71.23 & 0.79 & 69.43 & 0.86 & 69.06 \\
\hline TV & 0.86 & 70.62 & 0.93 & 74.44 & 0.80 & 68.43 & 0.88 & 70.41 \\
\hline DIP-TV & 0.90 & 72.37 & 0.91 & 72.48 & 0.83 & 68.55 & 0.97 & 76.18 \\
\hline TomoDIP-TV & 0.91 & 73.15 & 0.94 & 76.01 & 0.86 & 69.41 & 0.95 & 73.98 \\
\hline
\end{tabular}

Table 1. Quantitative comparison of reconstruction qualities from noise-free projections (Best SSIM and PSNR results per number of projections are shown in bold).

\section{Discussion}

In computed tomography, uniform sampling around the object might not always be possible, creating the socalled missing-wedge problem. Reconstructions using the traditional methods such as filtered back-projection show elongation, streaking, and ghost tail artifacts. Regularizers can be employed to reduce the effects of these artifacts. An example is the TV method which incorporates total variation regularization into the back-projection operation based on preliminary information or assumptions on the object. Although this approach is successful at increasing the reconstruction quality, artifacts cannot be eliminated completely; see Fig. 6 . On the other hand, deep image priors (DIP) architecture demonstrates that the whole process could be learned by self-training the network. The convolutional network employed becomes an "optimized regularizer" to reduce the artifacts. Using this idea, we have implemented a DIP network to reconstruct the 3D object directly from the projections. To incorporate total variation regularization, we applied self-training in an ADMM framework, which is able to decompose the problem into more manageable sub-problems. The resulting reconstructions showed that DIPTV approach increased the reconstruction quality notably, eliminating both missing wedge and noise artifacts (see Figs. 6 and 7).

Although we have shown that the DIP-TV method improves the reconstruction of 3D objects compared to the methods in the literature, the idea of the DIP is to regularize an existing solution. In DIP computed tomography reconstructions, the convolutional network is responsible for the inverse projection operation in addition 

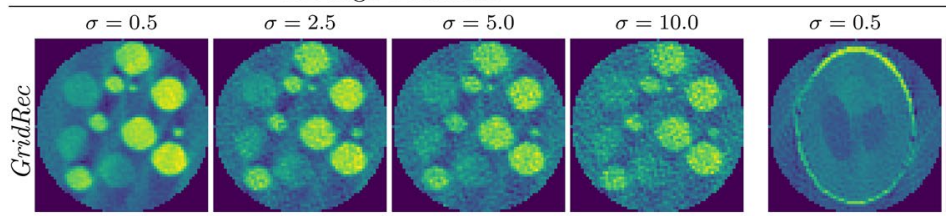

Shepp-Logan Phantom
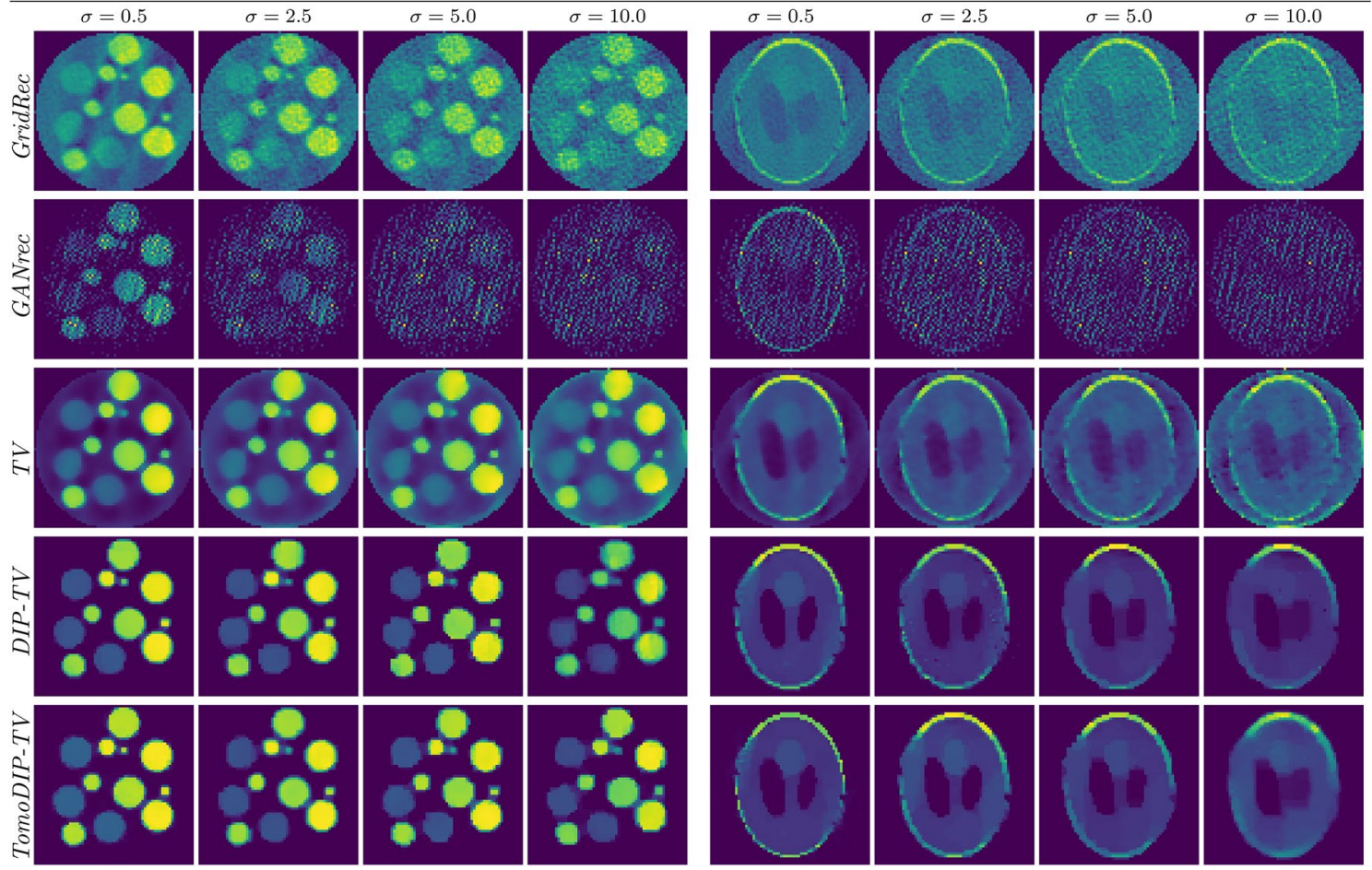

Figure 7. Reconstruction comparison for the XDesign phantom and the Shepp-Logan phantom for different noise levels and for projections between $0^{\circ}$ and $150^{\circ}$.

\begin{tabular}{|c|c|c|c|c|c|c|c|c|c|c|c|c|c|c|c|c|}
\hline \multirow[b]{3}{*}{ 3D noisy reconstruction } & \multicolumn{8}{|c|}{ XDesign phantom } & \multicolumn{8}{|c|}{ Shepp-Logan phantom } \\
\hline & \multicolumn{2}{|c|}{$\sigma^{2}=0.5$} & \multicolumn{2}{|c|}{$\sigma^{2}=2.5$} & \multicolumn{2}{|c|}{$\sigma^{2}=5$} & \multicolumn{2}{|c|}{$\sigma^{2}=10$} & \multicolumn{2}{|c|}{$\sigma^{2}=0.5$} & \multicolumn{2}{|c|}{$\sigma^{2}=2.5$} & \multicolumn{2}{|c|}{$\sigma^{2}=5$} & \multicolumn{2}{|c|}{$\sigma^{2}=10$} \\
\hline & ssim & psnr & ssim & psnr & ssim & psnr & ssim & psnr & ssim & psnr & ssim & psnr & ssim & psnr & ssim & psnr \\
\hline GridRec & 0.55 & 62.30 & 0.52 & 61.98 & 0.49 & 61.62 & 0.46 & 61.06 & 0.39 & 62.83 & 0.34 & 62.34 & 0.31 & 61.43 & 0.29 & 61.12 \\
\hline GANrec & 0.49 & 61.89 & 0.30 & 59.56 & 0.25 & 58.90 & 0.24 & 58.75 & 0.38 & 64.25 & 0.26 & 62.55 & 0.24 & 62.22 & 0.22 & 61.98 \\
\hline TV & 0.79 & 71.97 & 0.73 & 69.06 & 0.69 & 67.48 & 0.63 & 65.38 & 0.64 & 68.90 & 0.54 & 67.49 & 0.50 & 66.77 & 0.45 & 65.60 \\
\hline DIP-TV & 0.89 & 69.01 & 0.84 & 67.52 & 0.83 & 66.70 & 0.73 & 71.11 & 0.85 & 69.02 & 0.79 & 67.80 & 0.75 & 66.87 & 0.71 & 66.39 \\
\hline TomoDIP-TV & 0.97 & 75.11 & \begin{tabular}{|l|}
0.92 \\
\end{tabular} & \begin{tabular}{|l|}
72.91 \\
\end{tabular} & \begin{tabular}{|l|l}
0.90 \\
\end{tabular} & \begin{tabular}{|l|l|}
70.48 \\
\end{tabular} & \begin{tabular}{|l|}
0.81 \\
\end{tabular} & \begin{tabular}{|l|l}
66.82 \\
\end{tabular} & \begin{tabular}{|l|}
0.87 \\
\end{tabular} & \begin{tabular}{|l|l|}
69.81 \\
\end{tabular} & \begin{tabular}{|l|}
0.81 \\
\end{tabular} & \begin{tabular}{|l|l}
68.10 \\
\end{tabular} & \begin{tabular}{|l|}
0.77 \\
\end{tabular} & \begin{tabular}{|l|l}
67.34 \\
\end{tabular} & \begin{tabular}{|l|l|}
0.71 \\
\end{tabular} & $\mid 66.42$ \\
\hline
\end{tabular}

Table 2. Quantitative comparison of reconstruction qualities from noisy projections between $0^{\circ}$ and $150^{\circ}$ (Best SSIM and PSNR results per noise level are shown in bold).

to being a regularizer. However, inverse projection is a mapping operation, and it is shown that it can be modeled with a fully connected network more accurately. Thus, we have designed a cascaded network consisting of fully connected and convolutional layers as shown in Fig. 2, and replaced the fully convolutional network with it. Although having a smaller regularizer network, Figs. 6, 7, and Tables 1, 2 show that there is a considerable improvement in the reconstructions with the proposed method. With a more accurate modeling of the inverse operation, it can be seen that edges are more well-defined, and variation of reconstructed values is less for the proposed approach.

Despite the improved reconstruction quality, reconstruction time is a significant drawback for the proposed method. The method is a self-training algorithm, and it requires the network to be trained for each unique reconstruction, making the proposed method considerably slower compared to the supervised learning methods or the iterative reconstruction methods. For the simulations, the training is done on Nvidia GeForce RTX 2080 Super graphics card, where each inner ADMM iteration took approximately $0.37 \mathrm{~s}$, resulting in 431 min to reconstruct an object on average. The reconstruction time increases linearly with the increased number of voxels to be reconstructed, meaning an exponential increase for increased size in one dimension. This requires the size of the object to be limited for reasonable reconstruction times. However, experimental trials on using transfer learning applications showed that there is a possibility of reconstructing similar objects with the trained networks by minimal additional training, i.e., transfer learning, which can lower the reconstruction time up to 20-100 times. Starting the self-training with the optimized weights of the network from a previous reconstruction leads to a need for a smaller change in the network weights, which reduces the reconstruction time significantly. The temporal evaluation of the weights of the network in a previous reconstruction would mean a better starting 
(a) Original Sinogram

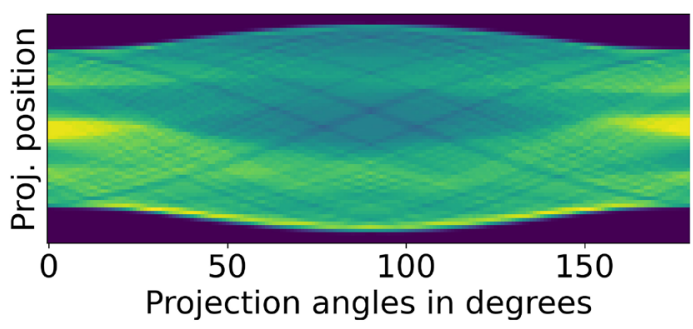

(b) Reconstructed Sinogram

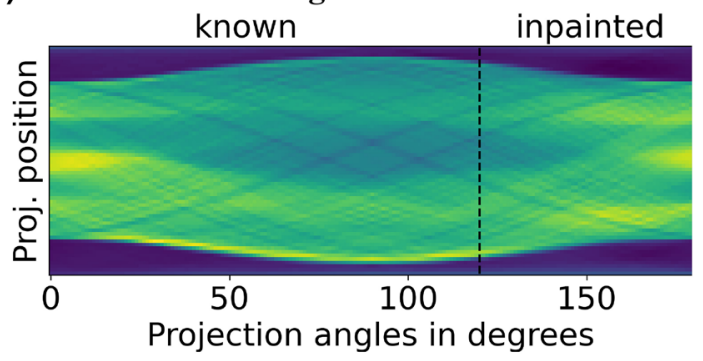

(c) Relative Error

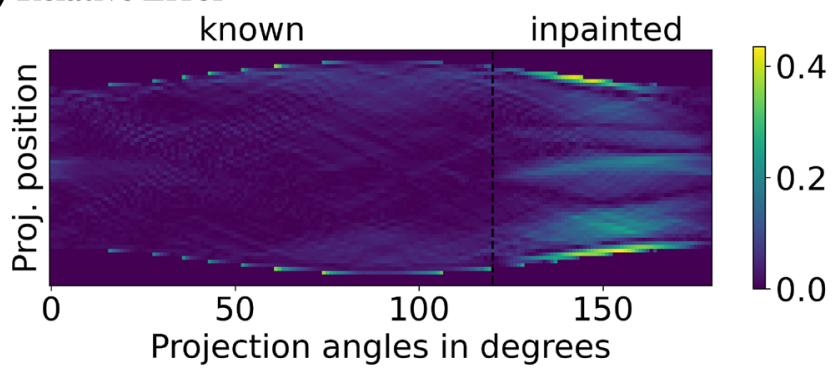

Figure 8. Reconstructed sinogram for noise-free $0^{\circ}-120^{\circ}$ projections, the sinogram from the original object for Shepp-Logan phantom, and the relative error between them.

point, and only a fine-tuning would be needed requiring a smaller amount of time. Also, the memory of GPUs is limited, and data transfers to and from the GPUs can become the bottleneck for self-training. The best solution to overcome these bottlenecks is to share the computational burden among multiple GPUs, which would increase the reconstruction speed and increase the possible size of the object to be reconstructed at the same time $e^{37,38}$.

\section{Conclusion}

In this paper, we have proposed a physics-based deep image priors application on a limited-angle tomography problem without the need for training data. We have shown that by separating the reconstruction problem using the alternating direction method of multipliers framework, we can augment regularization for prior information in the deep image priors network. First, we have demonstrated that deep image priors can improve the reconstruction quality with a trade-off between the reconstruction accuracy and the reconstruction time. Then, we have proposed a cascaded architecture network to model the inverse tomography problem more accurately and demonstrated that performance could be further increased. Besides, we have shown that an improved reconstruction quality for data with high noise variance is possible using the proposed algorithm.

\section{Data and code availability}

The data and the code used in this study are available upon request.

Received: 18 April 2021; Accepted: 17 August 2021

Published online: 06 September 2021

\section{References}

1. Frank, J. Three-Dimensional Electron Microscopy of Macromolecular Assemblies (Oxford University Press, 2006).

2. Nogales, E. The development of cryo-EM into a mainstream structural biology technique. Nat. Methods 13, 24-27. https://doi. org/10.1038/nmeth.3694 (2015).

3. Goris, B. et al. Measuring lattice strain in three dimensions through electron microscopy. Nano Lett. 15, 6996-7001. https://doi. org/10.1021/acs.nanolett.5b03008 (2015).

4. Miao, J., Ercius, P. \& Billinge, S. J. L. Atomic electron tomography: 3d structures without crystals. Science 353, aaf2157. https://doi. org/10.1126/science.aaf2157 (2016).

5. Yang, Y. et al. Deciphering chemical order/disorder and material properties at the single-atom level. Nature 542, 75-79. https:// doi.org/10.1038/nature21042 (2017).

6. Ardila, D. et al. End-to-end lung cancer screening with three-dimensional deep learning on low-dose chest computed tomography. Nat. Med. 25, 954-961. https://doi.org/10.1038/s41591-019-0447-x (2019).

7. Nikitin, V. et al. Photon-limited ptychography of 3d objects via Bayesian reconstruction. OSA Contin. 2, 2948. https://doi.org/10. 1364/osac.2.002948 (2019).

8. Midgley, P. \& Weyland, M. 3D Electron microscopy in the physical sciences: The development of z-contrast and EFTEM tomography. Ultramicroscopy 96, 413-431. https://doi.org/10.1016/s0304-3991(03)00105-0 (2003).

9. Gordon, R., Bender, R. \& Herman, G. T. Algebraic reconstruction techniques (ART) for three-dimensional electron microscopy and x-ray photography. J. Theor. Biol. 29, 471-481. https://doi.org/10.1016/0022-5193(70)90109-8 (1970).

10. Gilbert, P. Iterative methods for the three-dimensional reconstruction of an object from projections. J. Theor. Biol. 36, $105-117$. https://doi.org/10.1016/0022-5193(72)90180-4 (1972). 
11. Candès, E. J., Romberg, J. \& Tao, T. Robust uncertainty principles: Exact signal reconstruction from highly incomplete frequency information. IEEE Trans. Inf. Theory 52, 489-509 (2006).

12. Donoho, D. L. Compressed sensing. IEEE Trans. Inf. Theory 52, 1289-1306 (2006).

13. Ghani, M. U. \& Karl, W. C. Deep learning-based sinogram completion for low-dose CT. In 2018 IEEE 13th Image, Video, and Multidimensional Signal Processing Workshop (IVMSP). https://doi.org/10.1109/ivmspw.2018.8448403 (IEEE, 2018).

14. Yoo, S., Yang, X., Wolfman, M., Gürsoy, D. \& Katsaggelos, A. K. Sinogram image completion for limited angle tomography with generative adversarial networks. In 2019 IEEE International Conference on Image Processing (ICIP), 1252-1256 (IEEE, 2019).

15. Ding, G., Liu, Y., Zhang, R. \& Xin, H. L. A joint deep learning model to recover information and reduce artifacts in missing-wedge sinograms for electron tomography and beyond. Sci. Rep.https://doi.org/10.1038/s41598-019-49267-x (2019).

16. Anirudh, R. et al. Lose the views: Limited angle CT reconstruction via implicit sinogram completion. In 2018 IEEE/CVF Conference on Computer Vision and Pattern Recognition. https://doi.org/10.1109/cvpr.2018.00664 (IEEE, 2018).

17. Lucas, A., Iliadis, M., Molina, R. \& Katsaggelos, A. K. Using deep neural networks for inverse problems in imaging: Beyond analytical methods. IEEE Signal Process. Mag. 35, 20-36. https://doi.org/10.1109/msp.2017.2760358 (2018).

18. Wang, J., Zeng, L., Wang, C. \& Guo, Y. ADMM-based deep reconstruction for limited-angle CT. Phys. Med. Biol. 64, 115011. https:// doi.org/10.1088/1361-6560/ablaba (2019).

19. Ulyanov, D., Vedaldi, A. \& Lempitsky, V. Deep image prior. Int. J. Comput. Vis. 128, 1867-1888. https://doi.org/10.1007/s11263020-01303-4 (2020).

20. Baguer, D. O., Leuschner, J. \& Schmidt, M. Computed tomography reconstruction using deep image prior and learned reconstruction methods. Inverse Probl. 36, 094004. https://doi.org/10.1088/1361-6420/aba415 (2020).

21. Zhou, K. C. \& Horstmeyer, R. Diffraction tomography with a deep image prior. Opt. Exp. 28, 12872. https://doi.org/10.1364/oe. $379200(2020)$

22. Yang, X. et al. Tomographic reconstruction with a generative adversarial network. J. Synchrotron Radiat. 27, 486-493. https://doi. org/10.1107/s1600577520000831 (2020).

23. Ching, D. J. \& Gürsoy, D. XDesign: An Open-source Software Package for Designing X-ray Imaging Phantoms and Experiments. https://github.com/tomography/xdesign.

24. Boyd, S., Parikh, N., Chu, E., Peleato, B. \& Eckstein, J. Distributed optimization and statistical learning via the alternating direction method of multipliers. Found. Trends Mach. Learn. 3, 1-122 (2011).

25. Kingma, D. \& Ba, J. Adam: A method for stochastic optimization. In International Conference on Learning Representations (2014).

26. Donoho, D. De-noising by soft-thresholding. IEEE Trans. Inf. Theory 41, 613-627. https://doi.org/10.1109/18.382009 (1995).

27. Ba, J. L., Kiros, J. R. \& Hinton, G. E. Layer Normalization 1607, 06450 (2016).

28. Gürsoy, D., Carlo, F. D., Xiao, X. \& Jacobsen, C. TomoPy: A Framework for the Analysis of Synchrotron tomographic Data. https:// github.com/tomopy/tomopy.

29. Ching, D. J. \& Gürsoy, D. XDesign: An open-source software package for designing x-ray imaging phantoms and experiments. J. Synchrotron Radiat. 24, 537-544. https://doi.org/10.1107/s1600577517001928 (2017).

30. Gürsoy, D., Carlo, F. D., Xiao, X. \& Jacobsen, C. TomoPy: A framework for the analysis of synchrotron tomographic data. J. Synchrotron Radiat. 21, 1188-1193. https://doi.org/10.1107/s1600577514013939 (2014).

31. Dowd, B. A. et al. Developments in synchrotron x-ray computed microtomography at the National Synchrotron Light Source. In Bonse, U. (ed.) Developments in X-Ray Tomography II, vol. 3772, 224-236. https://doi.org/10.1117/12.363725. International Society for Optics and Photonics (SPIE, 1999).

32. Marone, F. \& Stampanoni, M. Regridding reconstruction algorithm for real-time tomographic imaging. J. Synchrotron Radiat. 19, 1029-1037. https://doi.org/10.1107/s0909049512032864 (2012).

33. Herman, G. T. Fundamentals of Computerized Tomography (Springer, London, 2009).

34. Goris, B., den Broek, W. V., Batenburg, K., Mezerji, H. H. \& Bals, S. Electron tomography based on a total variation minimization reconstruction technique. Ultramicroscopy 113, 120-130. https://doi.org/10.1016/j.ultramic.2011.11.004 (2012).

35. Biguri, A., Dosanjh, M., Hancock, S. \& Soleimani, M. TIGRE: A MATLAB-GPU toolbox for CBCT image reconstruction. Biomed. Phys. Eng. Exp. 2, 055010. https://doi.org/10.1088/2057-1976/2/5/055010 (2016).

36. Ronchetti, M. Torchradon: Fast Differentiable Routines for Computed Tomography. arXiv:2009.14788 (2020).

37. Hidayetoğlu, M. et al. MemXCT. In Proceedings of the International Conference for High Performance Computing, Networking, Storage and Analysis. https://doi.org/10.1145/3295500.3356220 (ACM, 2019).

38. Majchrowicz, M., Kapusta, P., Jackowska-Strumiłło, L., Banasiak, R. \& Sankowski, D. Multi-GPU, multi-node algorithms for acceleration of image reconstruction in $3 \mathrm{~d}$ electrical capacitance tomography in heterogeneous distributed system. Sensors $\mathbf{2 0}$, 391. https://doi.org/10.3390/s20020391 (2020).

\section{Acknowledgements}

The authors would like to thank Dr. Xiaogang Yang for providing assistance for implementation of GANrec method. This research used resources of the Advanced Photon Source, a U.S. Department of Energy (DOE) Office of Science User Facility, operated for the DOE Office of Science by Argonne National Laboratory under Contract No. DE-AC02-06CH11357.

\section{Author contributions}

S.B. implemented the idea and conducted the simulations. S.B., S.A., A.K.K., and D.G. conceived the idea, analyzed the results, and wrote the manuscript. All authors reviewed and approved the final version of the manuscript.

\section{Competing interests}

The authors declare no competing interests.

\section{Additional information}

Supplementary Information The online version contains supplementary material available at https://doi.org/ 10.1038/s41598-021-97226-2.

Correspondence and requests for materials should be addressed to S.B.

Reprints and permissions information is available at www.nature.com/reprints.

Publisher's note Springer Nature remains neutral with regard to jurisdictional claims in published maps and institutional affiliations. 
(c) (i) Open Access This article is licensed under a Creative Commons Attribution 4.0 International cc) License, which permits use, sharing, adaptation, distribution and reproduction in any medium or format, as long as you give appropriate credit to the original author(s) and the source, provide a link to the Creative Commons licence, and indicate if changes were made. The images or other third party material in this article are included in the article's Creative Commons licence, unless indicated otherwise in a credit line to the material. If material is not included in the article's Creative Commons licence and your intended use is not permitted by statutory regulation or exceeds the permitted use, you will need to obtain permission directly from the copyright holder. To view a copy of this licence, visit http://creativecommons.org/licenses/by/4.0/.

(C) The Author(s) 2021 